matters arising

\section{Origin of cellular senescence}

I HOPE to clarify a point regarding the recent letter of Dykhuizen ${ }^{1}$. He speculated that cellular senescence is the result of a selection process imposed by an organism's need to ensure its survival by limiting the size of atherosclerotic plaques which, he believed, originated from relocated fibroblasts. This is an interesting new view of the limit on fibroblast doubling potential described by Hayflick ${ }^{2}$ and others, and the predictions he derives from the hypothesis can be tested experimentally. These include the ultimate cessation of plaque growth when cells at the edge senesce, higher division potential of cells isolated from the centres of plaques, and a correlation between final plaque size and the age of the organism at the plaque's initiation. The fulfilment of these predictions will shed some light on the (probably fibroblastic) origin of plaques but is not critically dependent on an evolved origin for cellular senescence (or doubling potential limit) in the manner Dykhuizen suggests.

The expected observations could be explained, without resort to an evolutionary origin, simply on the basis of known facts. Specifically, cellular senescence manifests itself through a limit on the doubling capacity of fibroblasts; such cells are subject to "contact inhibition" of division ${ }^{2}$, and the doubling potential of fibroblasts is related to the age of the donor ${ }^{3}$. Whatever the ultimate cause of cellular senescence or its origin, the behaviour, given these facts, will be the same if plaques do arise from fibroblasts.

The predictions thus do not differentiate between Dykhuizen's explanation of an evolved origin for cellular senescence and a number of other processes which have been postulated as the primary cause of ageing such as error accumulation, a specific genetic programme or the exhaustion of a developmental programme.

\section{Roy Widdus}

The Center for Theoretical Biology, State University of New York/Buffalo, 4248 Ridge Lea,

Amherst, New York 14226

1 Dykhuizen, D., Nature, 251, 616-618 (1974). 2 Hayflick, L., Expl Cell Res., 37, 614-636 (1965). Martin, G. M., Sprague, C. A., and Epstein, C. J.
Lab. Invest., 23, 86-92 (1970).

DYKhUIZEN REPLIES-The lack of an understandable selective advantage for cellular senescence has been a major criticism of a hypothesis for its evolutionary origin ${ }^{1}$. The main point of my paper $^{2}$ was to provide a reasonable evolutionary explanation. To do this, I presented a nevel theory of the formation of atherosclerotic plaques. The predictions were meant to test this theory which, if correct, imp'ies that the size of plaques is limited by cell senescence, providing a possible selective advantage to the organism.

Given the assumption that a specific genetic programme could only arise through evolution, the evolutionary origin of cell senescence could be demonstrated by finding a gene, which, when rendered non-functional, allows cells which would normally senesce to become immortal. The error and evolutionary hypotheses could possibly be distinguished by determining the percentage of daughter cells of recently divided cells which go on to divide in a senescent culture and in an immortal culture derived from otherwise similar cells. Orgel ${ }^{1}$ suggested that if his error theory holds, then both percentages should be near $50 \%$-slightly less in the senescent and slightly more in the immortal culture. If the hypothesis of evolutionary origin is correct, these percentages should be quite different, say, $20 \%$ and $90 \%$ respectively.

\section{Australian National University.}

1 Orgel, L. E., Nature, 243, 441-445 (1973). 2 Dykhuizen, D., Nature, 251, 616-618 (1974).

\section{Further note on electron diffraction symmetries}

IN connection with diffraction symmetries it has been suggested ${ }^{1}$ that there are two conditions for a centrosymmetric pattern though there is some contention that the first of these conditions is incomplete. As the chief protagonists in this debate we wish to clarify the situation with respect to zero-beam symmetry and reciprocity.

We shall consider first those symmetries observable in convergent-beam zoneaxis patterns, that is, restricted sections of reciprocal space distributions disposed on a plane with the geometry of the diffraction pattern. Under these restrictions, a rotation diad along the zone axis completely determines a centrosymmetric pattern for all beams, that is, it is a sufficient condition for the whole pattern and does not require any consideration of reciprocity. (Note that to preserve the diad symmetry consideration must be restricted to either parallel-sided foils lying perpendicular to the incident beam, or else to crystals with boundaries which conform to the diad symmetry.)

This covers the example given by
Steeds ${ }^{2}$ and requires a correction to the table presented by Goodman ${ }^{7}$. To the last column of entry (2) in the table should be added the words "unless there is a rotation diad parallel to the zone, in which case the pattern will be centrosymmetric". Consideration of reciprocity allows general conditions to be found for zero-beam symmetry which do not apply to the remainder of the pattern as a whole but also give the internal symmetry of each dark-field intensity distribution. Thus, a central mirror or glide plane (condition 1 of Goodman $^{1}$ ) is a sufficient condition for centrosymmetry in the zero-beam pattern, but not in the remainder of the pattern. For other symmetries, a rotation diad or twofold screw axis, midway between the surfaces and parallel to them, ensures a mirror line in the zero-beam pattern (though not in the remainder of the pattern), perpendicular to the diad or screw axis (though this does not ensure a centre of symmetry in the zero-beam pattern). Higher symmetries than a mirror line or a centre can be built up by combination. Derivation of these rules from the invariance properties of the crystal does not require an explicit solution for the wave function, and can be applied to a crystal of any structure or surface geometry.

From such rules one can proceed to derive a description of a zone-axis pattern for a particular space group. But considerations of electron diffraction always concern crystals that are finite in at least one direction, and the strong interaction of electrons with the crystal potential makes it impossible to ignore the boundary conditions, so the symmetry of the total crystal must, therefore, be considered. The influence of tilted surfaces may be avoided to any accuracy by choosing crystal foils sufficiently parallelsided and horizontal (say in the range of $0-20^{\circ}$ ). But the influence of crystal termination in a flat horizontal specimen will mean that the crystal may not have the three-dimensional symmetry of the space group, and in such cases the crystal boundaries used in a symmetry derivation need to be stated.

Experimental limitations need to be considered. Convergent-beam diffraction, and diffraction-contoured micrographs, are practical methods of symmetry analysis with individual limitations. The pattern symmetries can be expressed by two-dimensional point-group symbols, whether investigating two-dimensional projection symmetries or three-dimen- 